\title{
The opinion and experience of surgeons with laparoscopic bowel grasper haptics
}

\author{
Eleonora P. Westebring-van der Putten ${ }^{1}$, Margriet C. J. Berben ${ }^{1}$, Richard H. M. Goossens ${ }^{1}$, \\ Jack J. Jakimowicz ${ }^{1,2}$, Jenny Dankelman ${ }^{3}$
}

\footnotetext{
${ }^{1}$ Department of Applied Ergonomics and Design, Faculty of Industrial Design Engineering, Delft University of Technology, Delft, the Netherlands;

${ }^{2}$ Department of Surgery, Catharina Hospital, Eindhoven, the Netherlands;

${ }^{3}$ Department of Biomechanical Engineering, Faculty of Mechanical, Maritime and Materials Sciences, Delft University of Technology, Delft, the Netherlands.

Email: e.p.westebring-vanderputten@tudelft.nl
}

Received 17 December 2009; revised 28 December 2009; accepted 12 January 2010.

\section{ABSTRACT}

Background: In order to develop new and better laparoscopic bowel instruments, which reduces patient risks, the opinions and experience that surgeons have with current laparoscopic bowel grasper haptics is important. In this study we explored this by means of a questionnaire. Method: A total of 386 onlinequestionnaires, were sent to laparoscopic surgeons working in European hospitals. They were all members of the European Association of Endoscopic Surgery and perform laparoscopic obesities or bowel surgery. Surgeons where divided into different age and experience groups. Results: A total of 174 completely filled out forms were analyzed. In total, $16 \%$ of the surgeons cannot prevent damage when they pinch too hard, although they $(10 \%)$ might have seen or felt it. Seven percent of the respondents were not able to see or feel tissue slippage. Whereas $31 \%$ can see or feel slippage they cannot do anything to prevent it. Overall, most of the respondents would appreciate technical changes in the laparoscopic bowel graspers to reduce tissue damage. Of all the respondents, $79 \%$ maintain that it is necessary to have a new laparoscopic grasper with augmented feedback. The majority of the respondents $(\mathbf{7 7 \%})$ would like to have tactile feedback as an indication of the level of pinch force. There are not many differences in the opinions of surgeons at different skill levels. Conclusion: From the results of the questionnaire and the other comments made by respondents it is evident that research and developments in the field of new laparoscopic graspers should continue.

Keywords: Laparoscopy; Vision and Experience; Bowel Graspers; Questionnaire; Haptic Feedback

\section{INTRODUCTION}

Laparoscopic surgery has many benefits for the patient, such as fewer traumas, shorter hospital stays and reduced recovery times [1-5]. However, this technique gives rise to difficulties for the surgeon such as reduced haptics and indirect vision [6], which in turn may lead to a higher rate of adverse events [7]. During laparoscopic bowel surgery stress injury, which leads to tissue damage (e.g. perforation), pathological scar tissue formation, bleeding, adhesions, and loss of bowel motility may occur when the instrument is pinched with excessive force or when tissue slips from the grasper $[8,9]$.

Many studies are currently being performed to establish the best way of reducing tissue damage during laparoscopic procedures (for a review of this see [10]). One of our own projects concerns laparoscopic grasp control. There we are trying to determine whether augmented feedback in relation to excessive pinch force and tissue slippage during laparoscopic grasping may improve performance. Preliminary tests with augmented feedback containing grasp force information have shown that the accuracy/level of grasping forces has indeed increased. The main aim of the project is thus to find the best kind of augmented feedback in relation to grasp force during laparoscopic grasping. Laparoscopic obesities and bowel surgery is chosen as the applicable field, as the tissue of the bowel is very delicate. Good grasp control is therefore a prerequisite in the correct performance of bowel surgery.

Apart from gaining results from experiments, we are interested in surgeons' opinions and experience with the current laparoscopic graspers during bowel surgery. This way researcher can develop instruments that fulfill the demands and wishes of the surgeons who are going to use the instruments. Current literature does not provide 
us with information retrieved from large groups of surgeons. Individual surgeons are asked to provide research groups with their opinion and experiences, although, these opinions are useful they might not represent the opinion of the whole user group. The amount of research done in the field of improving haptics sugges that this need is obvious, however, this has not been confirmed by large user groups. To collect this information, we compiled a questionnaire, which was first approved by the technical committee of the European Association for Endoscopic Surgery (EAES). The questionnaire was distributed to surgeons who use laparoscopic techniques. It included questions on laparoscopic surgery in general, laparoscopic bowel surgery, (augmented) feedback on pinch force information during laparoscopic grasping, involvement in hospital innovation and awareness and participation in research projects devoted to augmented feedback. This article will present the findings of that questionnaire.

\section{METHODS}

In total, 386 surgeons from different European hospitals (members of the EAES who perform laparoscopic bowel and obesities surgery), were approached by email and asked to fill in a questionnaire via the Internet (developed using NETQuestionnaires 6.0).

Apart from the overall opinions of the whole group we were also interested to see whether there were differences in the answers given by surgeons of different ages or levels of experience. We therefore distinguished three categories based on experience in terms of number of operations, experience in terms of years and age. All the surgeons were divided into one of the four levels given within each category (see Table 1 in the Result section).

Table 1. Devision in groups of the 174 respondents.

\begin{tabular}{llc}
\hline & \multicolumn{2}{c}{ Respondents (\%) } \\
\hline & $<500$ & 30 \\
Experience (no. of operations) & $500-1000$ & 17 \\
& $1000-2000$ & 20 \\
& $>2000$ & 33 \\
& $<5$ & 9 \\
Experience (years) & $5-10$ & 22 \\
& $10-15$ & 28 \\
Age (years) & $15-20$ & 41 \\
& $<40$ & 6 \\
& $40-50$ & 26 \\
& $50-60$ & 40 \\
\hline
\end{tabular}

The collected data was exported and processed into SPSS 16.0 for Microsoft Windows XP. The questions asked can be found in the appendix. Most questions had a one-answer option. Questions 6, 7, 8 and 10 allowed several answers. With each question it was possible to give additional comment.

To make sure that each respondent used the same definitions, we used the following explanation for tactile and proprioceptive feedback. Tactile perception relates to the perception of pressure, vibration, and texture (also sometimes called discriminative touch or cutaneous sense), and relies on different receptors in the skin (cutaneous mechanoreceptors). Proprioception (haptics) concerns the perception of posture and the position of the limbs, body and head in space and their positioning relative to each other, including the vestibular system, cutaneous sense and kinesthesia [11].

\section{RESULTS AND DISCUSSION}

A total of 281 surgeons responded. Of the 281 respondents, 174 submitted a completely filled-in questionnaire. This resulted in a completely filled out rate of $45 \%$. The experience level of the surgeons ranged from 80 to more than 10.000 operations and from $<5$ to $15-20$ years of experience. The age of the surgeons ranged from 29 to 69 years. The amount of surgeons that responded is enough to make rough conclusions about their opinion. Although, each new development in this field should check its specific need with the user group. The results can be biased, as it is possible that the surgeons that did not fill the questionnaire are indifference for the topic.

\subsection{Results from the Complete Group of Respondents}

Forty-six percent of the respondents use grasper 1 to grasp bowel tissue, followed by 24\% who use grasper 2 . Graspers 3, 4 and 5 where used by 10,10 and $3 \%$ of the respondents respectively and only $7 \%$ of the respondents reported using another type of grasper. Grasper 1 was used in our previous studies [9,12,13]. The answers to this question confirmed that this choice of bowel grasper was suitable for representing the bowel graspers used in practice.

From Figure 2 it can been seen that $51 \%$ of the respondents can feel when they apply excessive pinch force to the tissue and are able to adjust the pinch force to prevent damage. In total, $33 \%$ of the respondents can see when they apply excessive pinch force and are able to prevent damage. Six percent of the respondents cannot see or feel when they apply excessive pinch force to the tissue. Finally $16 \%$ of the surgeons cannot prevent damage although $10 \%$ might see or feel it.

Figures 3 shows that 32\% of the respondents can feel, $30 \%$ can see tissue slippage and are able to prevent it. In total, seven percent of the respondents can not 


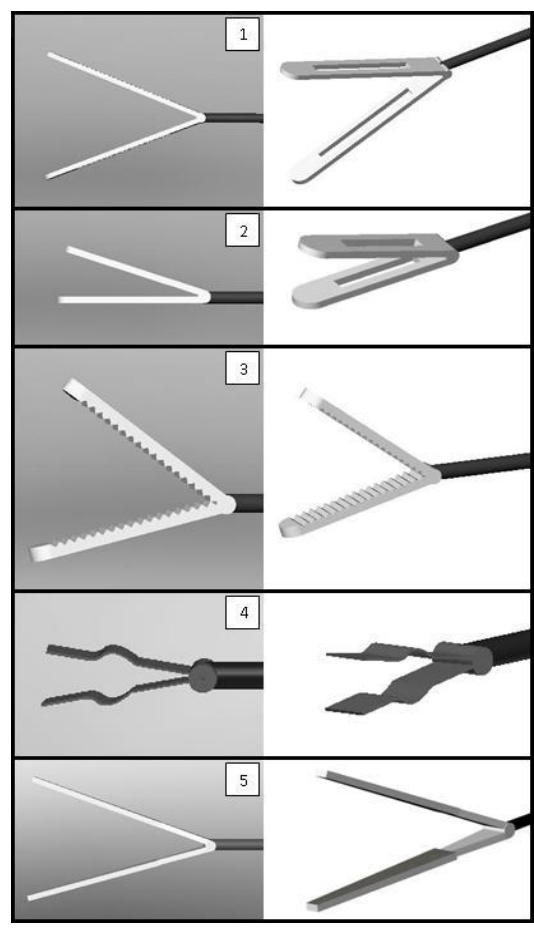

Figure 1. Images of laparoscopic graspers (see question 2). see or feel when tissue is about to slip. Some $31 \%$ of the respondents can see or feel slippage but they cannot do anything to prevent it. The results state that $94 \%$ of the respondents indicate that they notice tissue slippage, however, $38 \%$ of the respondents indicate that they cannot prevent it. These results show that there is a high percentage of the surgeons who cannot prevent tissue damage through slip. Heijnsdijk et al. [8] discovered during a study carried out during 10 laparoscopic colectomies and 15 cholecystectomics conducted by experienced surgeons that the bowel slipped out of the grasper in $7 \%$ of the grasp actions, whereas the gallbladder slipped out in $17 \%$ of cases. Thus, it seems that even experienced surgeons have difficulty maintaining an accurate pinch force.

In total, $32 \%$ of the respondents are aware of the existence of research projects linked to augmented feedback on pinch force information within laparoscopy and the respondents this questionnaire was the first time they heard anything about it. This could indicate that surgeons are not concerned about this issue. However, if we look at the questionnaire response rate, we see that surgeons are concerned. Further research into this topic is therefore important. In addition, the results of these studies should be made easily accessible to surgeons.

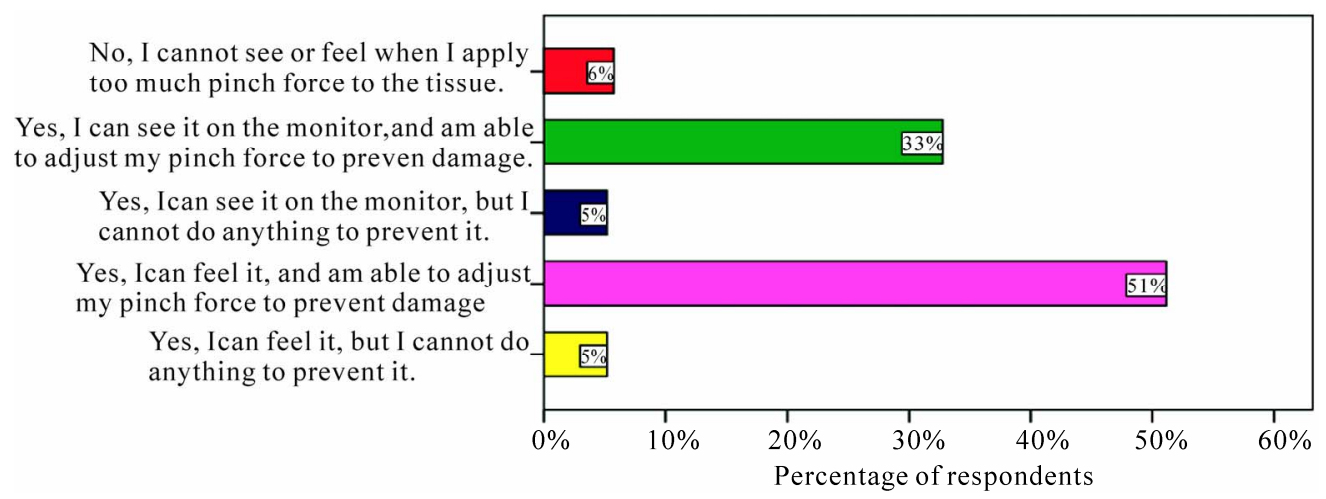

Figure 2. Respondent awareness of excessive pinch force usage. Answer on question 3. "Do you notice when you are about to apply too much pinch force on the tissue?”

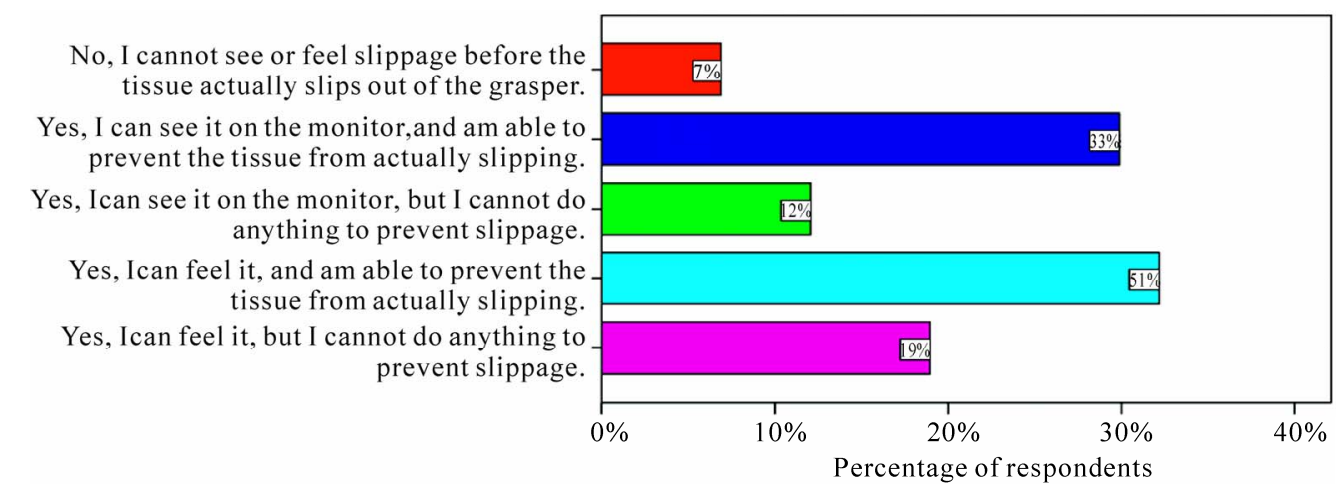

Figure 3. Respondent awareness of tissue slippage. Answer on question 4. "Do you notice when tissue is about to slip?” 
Ultimately $12 \%$ of the respondents had experience with some form of augmented feedback regarding pinch force within laparoscopy. Table 3 shows which form of augmented feedback these surgeons had experience with. Some respondents had experience with more than one form of augmented feedback. The majority used visual (95\%) or tactile feedback (81\%).

In total, $18 \%$ of the respondents had used a form of augmented feedback on pinch force information, during their virtual reality training. Table 4 shows the form of augmented feedback that the respondents used during virtual reality training. Some respondents had had experience with various forms of augmented feedback during their virtual reality training. However, the majority used visual or tactile feedback.

The results of questions six and seven show that tactile and visual augmented feedback is used in research much more frequently than audible and proprioceptive feedback. This can be explained by the fact that additional audible signals in the operating room will distract the surgeon, as there are so many other sounds already. Augmented proprioceptive feedback is technically more difficult to implement and it will be hard for the surgeon to interpret unless a natural reaction is provoked.

The questionnaire gave the respondents the opportunity to indicate their preferred augmented feedback form as an indication of the levels of pinch force. Figure 4 shows the preferences of the respondents. Most of the respondents would prefer to use tactile feedback as an indication of the level of pinch force (77\%), followed by visual feedback (39\%). Only $7 \%$ of the respondents do not like to use augmented feedback as an indication of the level of pinch force.
Table 3. The form of augmented feedback regarding pinch force that respondents had experienced within laparoscopy.

\begin{tabular}{lcc}
\hline $\begin{array}{c}\text { Form of additional } \\
\text { feedback }\end{array}$ & $\begin{array}{c}\text { Number of } \\
\text { respondents* }\end{array}$ & \% of respondents \\
\hline Visual feedback & 20 & 95 \\
Audible feedback & 0 & 0 \\
Tactile feedback & 17 & 81 \\
Proprioceptive feedback & 7 & 33 \\
Otherwise, (open response) & 0 & 0 \\
\hline
\end{tabular}

*Twelve percent of the total number or respondents answered question 6 with 'yes'. The number and percentage of respondents out of this twelve percent who used this form of augmented feedback during laparoscopy is indicated.

Note that some respondents had experienced multiple forms of augmented feedback.

Table 4. The form of augmented feedback regarding pinch force that respondents had experienced during virtual reality training.

\begin{tabular}{lcc}
\hline $\begin{array}{c}\text { Form of augmented } \\
\text { feedback }\end{array}$ & $\begin{array}{c}\text { Number of } \\
\text { respondents* }\end{array}$ & \% of respondents \\
\hline Visual feedback & 19 & 61 \\
Audible feedback & 6 & 19 \\
Tactile feedback & 19 & 61 \\
Proprioceptive feedback & 5 & 16 \\
Otherwise, (open response) & 2 & 6
\end{tabular}

*Eighteen percent of the total number or respondents answered question 6 with 'yes'. The number and percentage of respondents out of this eighteen percent who used this form of augmented feedback during laparoscopy is indicated.

Note that some respondents had experienced multiple forms of augmented feedback.

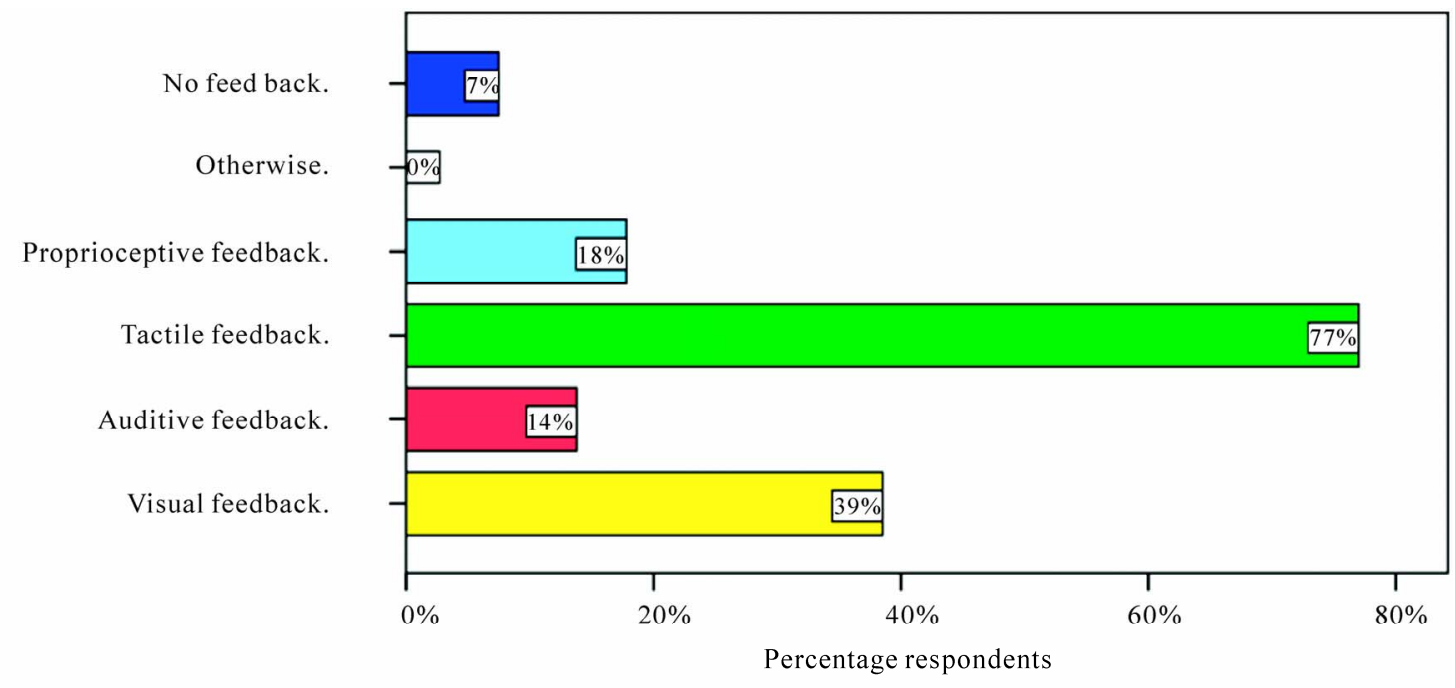

Figure 4. Preferred form of augmented feedback as indication of the levels of applied pinch force. Answer on question 8. "Which form of feedback would you like to use as an indication of the levels of pinch force ?” 
During laparoscopic procedures, 64\% of the respondents do not look at their hands while performing laparoscopic surgery, while 7\% do look several times, 9\% look frequently and $20 \%$ regularly look at their hands. When the respondents look at their hands, 30\% $(n=19)$ of them look at the hand position on the handle, $24 \%$ (n $=15)$ look at the fingers on the handle, $49 \%(n=31)$ look at the position of the handle and $21 \%(n=13)$ look at other things, for example the hand position relative to the abdomen, angle of instrument to the abdomen and the open or closed position of the handle. These results show that the handle is not the most suitable place to position a visual augmented feedback display but that it might be appropriate to have a tactile or proprioceptive display on the handle.

Twenty-one percent of the respondents have taken part in the modification and/or development of laparoscopic instruments. Twenty surgeons of that group (56\%) initiated the innovations themselves, and 25\% (9) of them indicated that a colleague instigated the developments. Manufactures were cited in 19\% (7) of cases as being responsible for the modification and/or development of laparoscopic instruments. None of the modifications or developments was enforced by the hospitals. This means that surgeons will use/develop new instruments when they are convinced of the added value with respect to the old instrument.

The respondents were asked if a new atraumatic grasper with additional haptic feedback is necessary. This question was answered with a 'yes' by $79 \%$ of the respondents. In their reply the respondents emphasized the safety of the grasper and the fact that it will prevent damage. The respondents who indicated that a new atraumatic grasper is not necessary are satisfied with the current laparoscopic instruments. Even though some of the respondents indicated that they notice when tissue is about to slip and that they can prevent it happening, 93 percent would like to have a laparoscopic instrument that provides some form of augmented feedback for slipping tissue. The conclusion therefore is that a new atraumatic grasper with augmented haptic feedback might help to reduce tissue damage.

Finally, 99\% of the respondents are open to technical changes in the field of laparoscopic instruments; only $1 \%$ of the respondents were not open to changes and indicated that the current laparoscopic instruments are fine. Should this research be continued, 95\% of the respondents declared themselves willing to participate in follow-up studies. This could mean that when a new laparoscopic instrument is introduced which contains augmented haptic feedback, a high proportion of the surgeons will want to use it.

\subsection{Results of the Categories}

There were no major differences in the response between the twelve groups (4 levels in each category). However, there were some minor differences and these are listed below.

Experienced surgeons (1000-2000 operations), use grasper $1(62 \%)$ more frequently than the less experienced ones (33\%). More experienced surgeons (> 2000 operations or 15-20 years of experience) indicate more frequently (63 and $61 \%$ respectively) than surgeons with less than 5 years of experience (19\%) that they can feel when they are applying excessive pinch force to the tissue. Regarding the use of visual verification to determine whether they are about to apply excessive pinch force, the difference between these categories is minimal. Less experienced surgeons find it more difficult to prevent tissue damage than experienced surgeons $(>2000$ operations, $15-20$ years experience and $>60$ age). This means that experience leads to a better interpretation of the task-intrinsic feedback. However, the learning curve for laparoscopic grasp control is long and even experienced surgeons do have difficulty using task-intrinsic feedback.

Surgeons of 60 years and older are not always the persons with the most experience in laparoscopic procedures. In contrast to the others, the category over the age of 60 does not have experience $(0 \%)$ with virtual reality training regarding augmented feedback on pinch force. This is probably due to the fact that these techniques did not exist when they where being educated. In addition, this category of surgeons looks more to their hands during any given procedure $(50 \%$ in this category as opposed to $30 \%$ in the categories $>2000$ operations or with $15-20$ years of experience). The last minor difference is that less experienced surgeons $(<500$ operations, $<5$ years experience or $<40$ years) indicate not having been involved in new developments compared to the other categories. This is obviously attributable to the fact that less experienced surgeons might think they do not have enough experience to innovate change.

\section{CONCLUSIONS}

The aim of this study was to estimate the opinions and experiences of surgeons with the use of laparoscopic bowel graspers from the point of view of haptics, Thanks to the large number of respondent's research and development of new instruments can now address the needs of the surgeons themselves.

In $38 \%$ of the cases the damage, according to the respondents, emanates from slip and in $16 \%$ of cases damage is attributable to excessive pinch force. This kind of tissue damage has to be reduced, possibly by using a laparoscopic instrument with augmented feedback on the levels of pinch force. The outcome of this study indicates a clear need for research and for the development of a new instrument with augmented feedback on force information and slippage. 


\section{REFERENCES}

[1] Cuschieri, A. (1995) Whither minimal access surgery: Tribulations and expectations. American Journal of Surgery, 169, 9-19.

[2] Moreno-Egea, A., Torralba, J., Morales, G., Fernandez, T., Guzman, P., Hita, G., Girela, E., Corral, M., Campillo, A. and Aguayo, J. (2005) Laparoscopic repair of secondary lumbar hernias: Open vs. laparoscopic surgery. A prospective, nonrandomized study. Cirugia Espanola, 77, 159-162.

[3] Dedemadi, G., Sgourakis, G., Karaliotas, C., Christofides, T., Kouraklis, G. and Karaliotas, C. (2006) Comparison of laparoscopic and open tension-free repair of recurrent inguinal hernias: A prospective randomized study. Surgical Endoscopy, 20, 1099-1104.

[4] Roumm, A., Pizzi, L., Goldfarb, N. and Cohn, H. (2005) Minimally invasive: Minimally reimbursed? An examination of six laparoscopic surgical procedures. Surgical Innovations, 12, 261-287.

[5] Stefanoni, M., Casciola, L., Ceccarelli, G., Spaziani, A., Conti, D., Bartoli, A., Zitti, L.D., Bellocchi, R. and Valeri, R. (2006) The biliopancreatic diversion. A comparison of laparoscopic and laparotomic techniques. Minerva Chirurgica, 61, 205-213.

[6] Stassen, H.G., Dankelman, J., Grimbergen, C.A. and Meijer, D.W. (2001) Man-machine aspects of minimally invasive surgery. Annual Reviews in Control, 25, 111-122.

[7] Dankelman, J., Wentink, M. and Stassen, H.G. and Gouma,
D.J. (2003) Human reliability and training in minimally invasive surgery. Minimally Invasive Therapy and Allied Technologies, 12, 129-135.

[8] Heijnsdijk, E.A.M., Dankelman, J. and Gouma, D.J. (2002) Effectiveness of grasping and duration of clamping using laparoscopic graspers. Surgical Endoscopy, 16, 1329-1331.

[9] Westebring-van der Putten, E.P., van den Dobbelsteen, J.J., Goossens, R.H., Jakimowicz, J.J. and Dankelman, J. (2009) Force feedback requirements for efficient laparoscopic grasp control. Ergonomics, 52, 1055-1066.

[10] Westebring-van der Putten, E.P., Goossens, R.H.M., Jakimowicz, J.J. and Dankelman, J. (2008) Haptics in minimally invasive surgery-a review. Minimally Invasive Therapy and Allied Technologies, 17, 3-16.

[11] Widmaier, E.P., Hershel, R. and Strang, K.T. (2004) Vander, Sherman, and Lucano's Human Physiology, The mechanisms of body function, 9th Edition, McGraw-Hill, 207266.

[12] Westebring-van der Putten, E.P., van den Dobbelsteen, J.J., Goossens, R.H.M., Jakimowicz, J.J. and Dankelman, J. (2009) Effect of laparoscopic grasper force transmission ratio on grasp control. Surgical Endoscopy, 23, 818-824.

[13] Westebring-van der Putten, E.P., Lysen, W.W., Hensen, V.D., Koopmans, N., Goossen, R.H.M., van den Dobbelsteen, J.J., Dankelman, J. and Jakimowicz, J.J. (2009) Tactile Feedback exceeds visual feedback to display tissue slippage in a laparoscopic grasper, Ios Press, 142, 420-425. 


\section{Appendix: Questionaire}

Laparoscopic operations in general

1. How many years have you been using laparoscopic surgery?

2. Which of the pictures below best represents the laparoscopic grasper you use to grasp bowel tissue ? The pictures are presented in Figure 1.

3. Do you notice when you are about to apply too much pinch force on the tissue?

- Yes, I can feel it, but I cannot do anything to prevent it.

- Yes, I can feel it, and am able to adjust my pinch force to prevent damage.

- Yes, I can see it on the monitor, but I cannot do anything to prevent it.

- Yes, I can see it on the monitor, and am able to adjust my pinch force to prevent damage.

- No, I cannot see or feel it when I apply too much pinch force to the tissue.

4. Do you notice when tissue is about to slip?

- Yes, I can feel it, but I cannot do anything to prevent slippage.

- Yes I can feel it, and am able to prevent the tissue from actually slipping.

- Yes, I can see it on the monitor, but I cannot do anything to prevent slippage.

- Yes, I can see it on the monitor, and am able to prevent the tissue from actually slipping.

- No, I cannot see or feel slippage before the tissue is actually out of the grasper.

Laparoscopic surgery and augmented feedback

5. Are you well informed on research into augmented feedback on pinch force information within laparoscopy?

- Yes, I have read studies.

- Yes, I take/took part in similar research.

- Yes, (open response).

- No, (open response).

The following definitions are used in questions 6,7 and 8: Tactile perception relates to the perception of pressure, vibration, and texture (also sometimes called discriminative touch or cutaneous sense), and relies on different receptors in the skin (cutaneous mechanoreceptors). Proprioception (haptics) concerns the perception of posture and the position of the limbs, body and head in space and their positioning relative to each other, including the vestibular system, cutaneous sense and kinesthesia

6. Do you have experience with a form of additional feedback regarding pinch force within laparoscopy?

- Yes, from another research project. This research is about:

- Visual feedback.

- Auditive feedback.

- Tactile feedback.

- Proprioceptive feedback.

- Otherwise, (open response).

- No.

7. Have you used a form of additional/alternative feedback on pinch force information, during a virtual reality training exercise?

- Yes, what kind of feedback have you used?

- Visual feedback.

- Auditive feedback.

- Tactile feedback.

- Proprioceptive feedback.

- Otherwise, (open response).

- No.

8. Which form of feedback would you like to use as an indication of the levels of pinch force?

-Visual feedback.

-Auditive feedback.

-Tactile feedback.

-Proprioceptive feedback

-Otherwise, (open response).

-No feedback.

9. Do you look at your hands while performing laparoscopic surgery?

- Yes, several times (once or twice every 10 minutes) during surgery.

- Yes, frequently (once or twice during every surgical procedure).

- Yes, regularly (but not during every surgical procedure).

- No, never. 
10. When you look at your hands what do you look at?

- My hand position on the handle.

- My fingers on the handle.

- The position of the handle.

- Otherwise, (open response).

Laparoscopic surgery and involvement

11. Have you taken part in the modification/development in laparoscopic instruments?

- Yes.

- No.

12. Who was responsible for the initiation of these changes?

- Self initiated.

- Instigated by a colleague.

- Enforced by the hospital.

- Enforced by the manufacturer.

13. Do you think a new atraumatic grasper with additional haptic feedback is necessary?

- Yes, because (open response).

- No, because (open response).

14. Are you open to technical changes in the field of laparoscopic instrumentation?

- Yes, I am open to changes and their applications.

- Yes, I am open to changes, will probably continue to use the current laparoscopic instruments.

- Yes, (open response).

- No, I am not open to changes, the current laparoscopic instruments are working fine.

- No, (open response).

15. Are you willing to take part in follow-up research, possibly including a test with a prototype?

- Yes, you may contact me in the future.

- No, I am not interested. 